\title{
Anal cancer: current and future treatment strategies
}

This article was published in the following Dove Press journal:

Gastrointestinal Cancer:Targets and Therapy

I6 January 201 I

Number of times this article has been viewed

\section{Joanna Y Chin \\ Theodore S Hong \\ Jennifer $Y$ Wo}

Department of Radiation Oncology, Massachusetts General Hospital, Boston, MA, USA
Correspondence: Jennifer $Y$ Wo Department of Radiation Oncology, Cox 3, Massachusetts General Hospital, 100 Blossom Street, Boston,

MA 02II4, USA

$\mathrm{Tel}+\mathrm{I} 6177267559$

Fax +l 6177263603

Email jwo@partners.org

\begin{abstract}
Anal cancer is a relatively rare malignancy, accounting for approximately $2 \%$ of gastrointestinal cancers. Concurrent chemoradiation with 5-fluorouracil/mitomycin remains the standard of care for the treatment of anal cancer. There is currently no proven role for platinum-based induction or adjuvant chemotherapy in anal cancer, even in cases of bulky disease. Multiple trials have shown that radiosensitization with concurrent chemotherapy is beneficial over radiation alone, and in particular, efforts to remove or substitute mitomycin from the chemoradiation regimen have been unsuccessful. Because local-regional control remains a challenge in the management of anal cancer, future studies will need to focus on radiation dose-escalation and/or addition of further chemotherapy or targeted agents. Patient selection, eg, with PET-CT or with biomarkers including HPV status, may be necessary to define patients who need more aggressive local treatment, ie, for patients with bulky disease, or to de-escalate treatment in others, ie, patients with early-stage, localized cancer.
\end{abstract}

Keywords: anal cancer, chemoradiation, IMRT

\section{Introduction to anal cancer}

Anal cancer is a relatively rare malignancy, accounting for approximately $2 \%$ of gastrointestinal cancers. Most patients present with rectal bleeding, pain, or sensation of an anal mass, and most disease is localized at diagnosis. Approximately $20 \%-40 \%$ of patients will present with lymph node involvement, and approximately $10 \%$ will present with metastatic disease. ${ }^{1}$

Organ preservation with definitive chemoradiation is the cornerstone of current treatment of anal cancer, with surgical resection reserved as salvage treatment for residual or recurrent disease. This management approach reflects a paradigm shift from the use of abdominoperineal resection (APR) as the primary mode of treatment, as was the case prior to the 1980 s. Surgery was associated with significant morbidity and historical 5-year survival rates of 50\%-60\% in early surgical series. ${ }^{2-4}$ Pioneering work in the 1970s and 1980s demonstrated survival rates at least equivalent to surgery with the use of pre-operative chemoradiation. ${ }^{5,6}$ When chemoradiation was shown to induce pathologic remission at planned APR, surgery was then reserved for salvage treatment, thereby avoiding permanent colostomy in the majority of cases. Though surgery and chemoradiation has not been compared directly in a prospective clinical trial, definitive chemoradiation has now become the standard of care in the treatment of anal cancer. ${ }^{7}$

Local control rates with combined modality therapy are $60 \%-90 \%$ in all tumor stages, and the sphincter is preserved in $65 \%$ of cases. ${ }^{2}$ High tumor stage, regional 
nodal involvement, and male gender are associated with worse prognosis. For example, the 5-year survival of inguinal or pelvic node-positive patients is approximately half of that of node-negative patients. ${ }^{3,4}$ The 5 -year relative survival rates are $70 \%-80 \%$ for localized anal cancer, $61 \%$ for regional disease, and $29 \%$ for M1 disease. ${ }^{1,8}$ Local failure continues to be a major challenge in the treatment of anal cancer. The use of radiation dose-escalation with intensity modulated radiation therapy and the use of targeted therapy are under investigation as potential tools to improve local and regional control.

\section{Epidemiology, etiology, and symptomology}

Anal cancer is a rare malignancy of the gastrointestinal tract, with an estimated 6230 new cancers in the United States in 2012. ${ }^{9}$ The incidence of anal cancer is increasing, however, particularly in urban populations and in patients diagnosed with HIV. ${ }^{10,11}$ In the US, the median age at diagnosis is 60-65. Women have a slightly higher incidence rate compared to men (1.79 per 100,000 women during 1973-2000, versus 1.55 per 100,000 men), particularly in the $\geq 50$ age group. In contrast, men have a higher incidence rate in the youngest age group (20-49 years). ${ }^{8}$ Risk factors include persistent human papillomavirus (HPV) infection, history of precancerous anal lesions such as condylomas or high-grade anal intraepithelial neoplasia, anoreceptive intercourse, female gender, and immunosuppression secondary to solid organ transplantation. The population at highest risk for anal cancer appears to be men who have sex with men (OR 17.3), ${ }^{12}$ and in particular in the subset of these men who are HIV-positive. An estimated $85 \%$ of invasive anal cancers are HPV-positive, predominantly HPV-16. ${ }^{13}$ Patients with HPV-associated anal cancer tend to present, on average, a decade younger than patients with HPV-negative cancer.

Though up to $20 \%$ of patients can be asymptomatic at presentation, most patients present with symptoms related to the mass, such as rectal bleeding, perineal pain, sensation of a mass at the anus, pruritis, and anal discharge. With proximal anal cancer tumors, patients may present with a change in bowel movements. Most patients are diagnosed with localized disease (eg, stage I-II), while 29\% have regional lymph node involvement and $8 \%-12 \%$ present with disease elsewhere. ${ }^{1}$ Metastases can be found in the liver, lung, bone, or subcutaneous tissue.

\section{Diagnostics and prevention}

Work-up requires a physical exam to assess for tumor location, size, and extent, as well as direct visualization of the mass with colonoscopy, flexible sigmoidoscopy, or rigid proctoscopy. Note should be made of sphincter function at diagnosis and an examination of the groins for lymph node involvement. In patients with significant pain, full examination may require anesthesia. CT scanning can be used to assess primary tumor invasion into surrounding organs and involvement of pelvic and inguinal lymph nodes. FDG PET-CT has also become part of the standard work-up, particularly to evaluate lymph nodes that are ambiguous on $\mathrm{CT}$, to aid in management as well as to serve as a pretreatment baseline. In women with suspected anal cancer, a full gynecologic exam must be performed to determine the extent of invasion into the posterior vagina, and to rule out other HPV-associated diseases such as cervical dysplasia. In patients with other risk factors or a history of multiple sexual partners, HIV testing is recommended.

Lymph nodes are involved in approximately $20 \%-40 \%$ of cases at the time of diagnosis. ${ }^{1}$ Masses above the anal verge drain first to the perirectal nodes, followed by the iliac lymph nodes; in contrast, lesions below the anal verge drain first to the inguinal lymph nodes. Suspicious lymph nodes should be evaluated by fine needle aspiration, if feasible and safe, to determine further management. A formal lymph node dissection is considered unnecessary due to delays in treatment and increases in morbidity (eg, leg edema).

PET scan is increasingly being used for its higher sensitivity in detecting regional nodal involvement for full staging prior to treatment. ${ }^{14}$ In one recent study using pre- and post-treatment PET scans in combination with anal biopsy and sentinel node biopsy findings in 53 patients, PET-CT was superior to CT in detecting the primary tumor $(89 \%$ versus $75 \%) .{ }^{15}$ PET-CT was positive for inguinal metastases in $23 \%$ of patients, and negative in $77 \%$ of patients; sentinel node biopsy confirmed the presence of inguinal metastases in only 8 of 41 patients, indicating a $10 \%$ false positive rate and a $5 \%$ false negative rate with PET-CT. Pre-treatment PET-CT upstaged $38 \%$ of patients and downstaged $25 \%$ of patients, leading to a revision of radiation fields in 5 patients. Furthermore, PET-CT was demonstrated to have clinical utility after treatment, leading to a change in management in $18 \%$ of anal cancer patients referred for PET-CT as part of their follow-up. ${ }^{16}$ Some of these changes included avoiding biopsy of post-radiotherapy scar tissue, deciding upon surgery for evidence of local recurrence, and changing management strategy from surgery to chemotherapy upon PET-CT confirmation of more extensive disease. In this study, PET-CT had a sensitivity of $93 \%$ and a specificity of $81 \%$. At the 3 month post-treatment follow-up in the above study by Mistrangelo et al, ${ }^{15}$ PET-CT had a sensitivity 
of $100 \%$, specificity of $97.4 \%$, positive predictive value of $66 \%$, and negative predictive value of $100 \%$. The high negative predictive value of PET-CT in the post-treatment setting is particularly useful in avoiding unnecessary biopsies of post-irradiated tissue.

Because of the high prevalence of HPV infection in patients with anal squamous cell carcinoma, there is interest in the use of the quadrivalent HPV vaccine in the prevention of anal cancer. It has been suggested that $80 \%$ of anal cancers can be avoided by HPV 16/18 vaccination. ${ }^{17,18}$ Men who have sex with men (MSM), in particular, are at higher risk than the general population in developing precancerous anal lesions as well as anal cancer irrespective of HIV or HPV status, and as such are potential candidates for HPV vaccination. In one study of 602 healthy MSM in the 16-26 age group, men randomized to receive the quadrivalent HPV vaccine had a lower rate of anal intraepithelial neoplasia (13 per 100 person-years) compared with the placebo group (17.5 per 100 person-years), and in particular, the rate of grade 2 or 3 anal intraepithelial neoplasia associated with oncogenic HPV infection was reduced by $54 \%$ in the vaccinated group. ${ }^{19}$ The vaccine was 94\% efficacious against persistent anal HPV-16, and $100 \%$ against persistent anal HPV-18 infection. The vaccine was also found to decrease the 2-year recurrence rate of precancerous high-grade anal intraepithelial neoplasia. ${ }^{20}$ Whether this translates into decreased incidence of anal cancer requires longer follow-up from vaccination.

\section{Current treatments}

Whereas surgical management was the first-line approach to treating anal cancer prior to the 1980 s, organ preservation with chemoradiation is now the mainstay of treatment following key studies by Dr Norman Nigro at Wayne State University in the 1970s. ${ }^{5,6}$ Surgery for anal cancer required an APR, and was associated with significant morbidity including permanent colostomy. The 5-year local recurrence and survival rates after radical excision were approximately $30 \%-50 \%$ and $40 \%-70 \%$, respectively, in several early surgical series. In 1974, Nigro, in an attempt to convert inoperable cases into surgical candidates, used combined chemoradiation with 5-fluorouracil (5-FU) and mitomycin-C (MMC) for patients with squamous cell cancer of the anus. ${ }^{5}$ Radiation dose by external beam was $30 \mathrm{~Gy}$. At the time of salvage surgery, three of the three patients in the case series showed pathologic complete response ( $\mathrm{pCR}$ ). In subsequent reports his group demonstrated pCR in 24 of 28 patients who received definitive treatment with chemoradiation. Followup showed that pCR translated into improved 5-year overall survival, and avoided APR, thereby preserving sphincter function, in $60 \%$ of the patients at 5 years. ${ }^{6}$

The necessity of chemotherapy in the Nigro regimen has been investigated in several randomized and non-randomized trials. One multicenter study, run by the United Kingdom Coordinating Committee on Cancer Research (UKCCCR), randomized patients to radiation alone or to a combined modality regimen of 5-FU and MMC during radiation (Table 1). ${ }^{21}$ The 3-year local failure rate was $50 \%$, with most failures occurring within 18 months of treatment (local failure, in this study, included surgeries for treatment-related morbidity). In the radiation alone arm, the local failure rate was $61 \%$, compared with $39 \%$ in the chemoradiation arm; furthermore, the proportion of patients requiring APR in the chemoradiation arm was halved relative to radiation alone $35 \%$ in the radiation alone arm, versus $17 \%$ with chemoradiation). Though there was a cancer specific survival advantage with chemoradiation over radiation alone, there was no 3-year overall survival difference between the two arms $(58 \%$ in patients receiving radiation alone, 65\% in concurrent chemoradiation arm, $P=0.25$ ). It is noteworthy that patients with M1 disease were not excluded in this study (albeit comprising only $3 \%$ of the study participants), and that males made up a higher percentage of the study population than in other randomized trials. As mentioned above, male gender has been demonstrated as a negative prognostic factor in several studies, and the higher male proportion in this study may account for the inferior local control and overall survival rates relative to similar trials in the same era.

The benefit of chemoradiation over radiation alone was also seen in patients with locally advanced disease, ie, T1-2 with nodal involvement or larger or more extensive cancers (T3-4) with or without nodal involvement, who were randomized in a European Organization for Research and Treatment of Cancer (EORTC) trial to radiotherapy alone or with chemoradiation with 5-FU and $\mathrm{MMC} .{ }^{22}$ Again, patients who received concurrent chemoradiation had a higher complete remission rate than patients who received radiation alone ( $80 \%$ vs $54 \%$, respectively), which translated into a $32 \%$ higher colostomyfree rate with chemoradiation. However, as in the UKCCCR trial, there was no difference in overall survival between the two study arms, nor in time to metastases.

Based on the UKCCCR, EORTC and other studies, the role of chemotherapy in addition to radiation for the management of anal cancer has been firmly established. Further studies have evaluated the choice of chemotherapy agents as radiosensitizers, in an attempt to improve on Nigro's original 5-FU and MMC regimen. Despite its known toxicity 


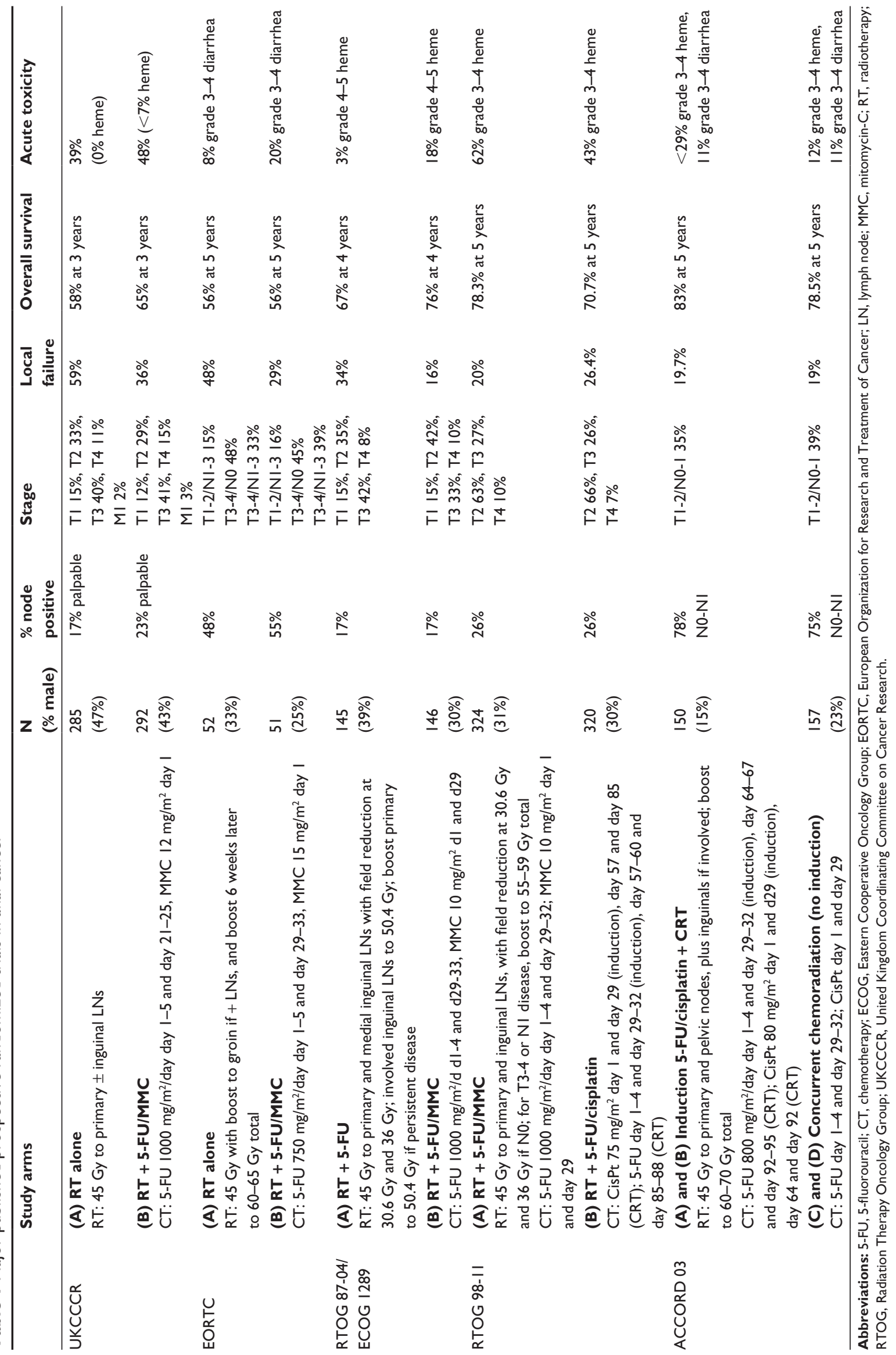


and relatively limited use outside of the management of anal cancer, attempts to remove or substitute $\mathrm{MMC}$ from the treatment of anal cancer have proven unsuccessful. The Radiation Therapy Oncology Group (RTOG) 87-04/ Eastern Cooperative Oncology Group (ECOG) 1289 trial compared the use of 5-FU alone versus 5-FU combined with MMC, in 310 patients with stage I-IIIA anal cancer undergoing definitive chemoradiation. ${ }^{23}$ Elimination of MMC resulted in almost doubling of 5-year local recurrence, an increase in colostomy rate $(23 \%$ versus $9 \%$ at 4 years, $P=0.002)$ and a decrease in 5 year disease free survival (from $64 \%$ in the $5-\mathrm{FU} / \mathrm{MMC}$ arm to $50 \%$ in the 5-FU alone arm, $P<0.003$ ). The colostomy rate reduction was greatest in patients with T3-4 disease, or in N0 patients. Despite increased Grade 4-5 toxicity in the 5 -FU/MMC arm $(23 \%$ vs $7 \%, P<0.001)$, primarily hematologic toxicity, there was no difference in overall survival in the two groups.

More recently, cisplatin has been studied as a substitute for MMC, based in part by cisplatin's documented efficacy in other disease sites, particularly in head and neck cancers. Initial support for the use of cisplatin instead of MMC came from several phase II studies. The ECOG E4292 study used concurrent 5-FU/cisplatin with a radiation dose of $59.4 \mathrm{~Gy}$ in 32 patients with localized anal cancer (the initial 19 patients had a planned treatment break after $36 \mathrm{~Gy}$, and subsequent participants had no planned break). ${ }^{24}$ Complete response rate in this group was $78 \%$, and an additional $19 \%$ had a partial response following chemoradiation. Five-year overall survival was $69 \%$, comparable to prior studies with concurrent chemoradiation. Similarly, Pieffert et $\mathrm{a}^{25}$ used 5-FU/cisplatin in the neoadjuvant setting and concurrent to radiation in 80 patients with anal cancer. This study showed good tolerance of the treatment regimen, and comparable complete remission, colostomy-free, and 3-year overall survival rates similar to historical data with chemoradiation. After treatment completion, which included four salvage APRs, all but five achieved complete remission. ${ }^{25}$ Another Phase II trial, ${ }^{26}$ run by the Cancer and Leukemia Group B, studied induction chemotherapy with $5-\mathrm{FU} /$ cisplatin followed by concurrent chemoradiation with $5-\mathrm{FU} / \mathrm{MMC}$ in high risk patients. High-risk patients were defined as having tumors $>5 \mathrm{~cm}$ in size, adjacent organ involvement, nodal size $>3 \mathrm{~cm}$, or any size tumor with bilateral nodal involvement. The premise of induction chemotherapy in these patients was to reduce tumor bulk prior to definitive chemoradiation for optimal response. In this study, $82 \%$ of 45 evaluable patients achieved a complete response, with $50 \%$ colostomy-free survival and $68 \%$ overall survival at 4 -year follow-up. ${ }^{26}$
Based on these Phase II data, RTOG 98-11 was designed to study whether cisplatin can be substituted for MMC in concurrent chemoradiation for anal cancer. ${ }^{27,28} \mathrm{Six}$ hundred eighty-two patients were randomized to receive either radiation with 5-FU/MMC, or to receive induction 5 -FU/cisplatin for 2 cycles followed by radiation with 5-FU/cisplatin. In the most recent update of the trial, the use of cisplatin was inferior to $\mathrm{MMC}$ in terms of both overall survival and disease-free survival (5-year disease-free survival: $67.8 \%$ vs $57.8 \%, P=0.006$; 5 -year overall survival: $78.3 \%$ vs $70.7 \%, P=0.026)$. An overall survival advantage of the 5-FU/MMC arm was observed despite an increase in grade 3-4 hematologic toxicity with the use of MMC. Late toxicity was not significantly different between the two arms. Based on this trial, it appears that MMC cannot be replaced with cisplatin in the treatment of anal cancer as the standard of care. Accordingly, a common chemotherapy regimen given concurrently with radiation in US practice is 5-FU $1000 \mathrm{mg} / \mathrm{m}^{2} /$ day continuously infused for 96 hours on days 1-4 and 29-32, along with mitomycin $12-15 \mathrm{mg} / \mathrm{m}^{2}$ if given as a single dose on day 1 , or at a lower dose if given over both chemotherapy cycles (ie, $10 \mathrm{mg} / \mathrm{m}^{2}$ on days 1 and 29).

It is worth emphasizing that the trial design of RTOG 98-11 does not permit direct comparison of cisplatin versus MMC, because patients in the 5-FU/cisplatin arm had a lead-in time to radiation of an additional 2 months compared with the 5-FU/MMC arm. In effect, this induction period with 5-FU/cisplatin clinically delayed the time to definitive treatment with chemoradiation. In fact, in RTOG 98-11, the median total treatment duration for the 5FU/cisplatin arm was 101 days, compared with a median of 49 days for the 5-FU/MMC arm. This more than doubled treatment time for the 5-FU/cisplatin arm despite the fact that more patients in the 5-FU/MMC needed a treatment break for treatment toxicity. The excess treatment duration time of the 5-FU/ cisplatin arm is important, because prior analyses suggest that overall increased treatment duration, independent of treatment regimen, negatively impacts overall survival, ${ }^{29,30}$ possibly due to accelerated repopulation during fractionated radiotherapy. This phenomenon has been shown in other disease sites as well.

In parallel with and since RTOG 98-11, other studies have also looked at substituting MMC with cisplatin. The ACT II trial in the UK compared the use of 5-FU/MMC versus 5-FU/cisplatin as radiosensitizers for anal cancer, without the potential confounder of induction chemotherapy. ${ }^{31,32}$ The trial included 940 patients, $62 \%$ of whom were male. Data presented in abstract form at the American Society of 
Clinical Oncology meeting in 2012, with a median follow-up of 5 years, shows similar rates of recurrence-free survival and overall survival between the two groups. Additionally, there is retrospective data from a single institution of 179 patients comparing chemoradiation with 5-FU/MMC versus 5-FU/cisplatin, showing similar rates of complete response $(73 \%$ in the cisplatin group versus $72 \%$ in the MMC group) and locoregional failure (35\% at 5 years in the cisplatin group versus $31 \%$ in the MMC group), even in the T3/T4 subgroup. ${ }^{33}$ There was equivalence between the two groups in overall survival, disease free survival, and in colostomy-free survival at 5 years. Reported rates of grade 2 or greater toxicities were similar regardless of chemotherapy agent, as was treatment duration. Therefore, both ACT II and the Brazil series suggest that 5-FU/cisplatin is not inferior to $5-\mathrm{FU} / \mathrm{MMC}$, and may be a reasonable alternative when MMC cannot be used.

Notably, the Intergroup/ACCORD03 phase III trial found no detriment to the use of induction chemotherapy in the treatment of anal cancer. ${ }^{34}$ The ACCORD03 trial used a $2 \times 2$ trial design comparing induction with 2 cycles of 5 -FU/cisplatin versus upfront chemoradiation, with a secondary randomization to a standard (15 Gy) or higher-dose (20-25 Gy) radiation boost. In both arms, chemoradiation used 5-FU/cisplatin in an administration schedule similar to the cisplatin arm of RTOG 98-11, and split-course radiation to $45 \mathrm{~Gy}$ in 25 fractions. Data from this trial suggests that neither induction chemotherapy nor a higher radiation boost leads to a colostomy-free survival advantage $(76.5 \%$ at 5 years with induction, compared with $75 \%$ in the group without induction) nor a tumor-free survival advantage (71.5\% with induction versus $64.8 \%$ without). ${ }^{34}$ This study did not separately analyze the role of induction chemotherapy in patients with advanced tumors, which as a subgroup may derive more benefit from treatment intensification with induction chemotherapy and/or radiation boost. In fact, $37 \%$ of the patients enrolled had early stage disease (T1-T2/N0-1), compared with 64\% with American Joint Committee on Cancer stage I-II disease in the cisplatin arm of RTOG 98-11. Notably, the rate of acute grade 3 or 4 hematologic toxicity was lower in the induction arms of the ACCORD03 trial (29\%) than in the 5-FU/cisplatin arm of RTOG 98-11 (42\%), despite similar chemotherapy schedules. Lower toxicity rates in the ACCORD03 trial may have contributed to improved disease-free survival and colostomy-free survival compared with RTOG 98-11 (57.8\% and $65 \%$ at 5 years, respectively, in the cisplatin arm of RTOG 98-11).
In addition to neoadjuvant chemotherapy, adjuvant chemotherapy has also been studied in the management of anal cancer. The ACT II trial mentioned above, in fact, includes a secondary randomization of adjuvant chemotherapy with two cycles of 5-FU/cisplatin, or observation alone, following definitive chemoradiation. At the 26-week follow-up, there was no difference in complete remission rate in patients receiving adjuvant chemotherapy versus observation alone ( $85 \%$ vs $82 \%$, respectively, $P=0.34){ }^{31,32}$

Patients with bulky disease, that is, with tumors over $5 \mathrm{~cm}$ in size and/or node positive disease, appear to fare worse in terms of disease-free survival, on multivariate analysis in RTOG 98-11. Their local-regional recurrence rate of $40 \%-64 \%$ is significantly higher than in patients with $\mathrm{T} 2 \mathrm{~N} 0$ or $\mathrm{T} 3 \mathrm{~N} 0$ disease $(\sim 20 \%)$. The very high rate of local-regional recurrence in patients with bulky disease following concurrent chemoradiation suggests that more aggressive treatment may be necessary in these patients, eg, minimizing treatment gaps, radiation dose escalation or adoption of a lower threshold for planned or early surgical resection. Additionally, the high rate of local-regional failure documented in patients with bulky disease in RTOG 98-11 demonstrates the need to treat pelvic and inguinal lymph nodes in these patients. As noted above, PET-CT is useful to more accurately assess nodal status during radiation planning.

Just as all patients with suspected anal cancer should undergo evaluation in a multidisciplinary setting, patients should also be in the care of a multidisciplinary team during treatment and in long-term follow-up. Acute and long-term dermatologic, hematologic, gastrointestinal, and genitourinary toxicities need to be assessed, and supportive care is provided with the goal to minimize treatment breaks if possible. Follow-up after treatment completion includes digital rectal exam with possible anoscopy; in addition, as discussed above, PET-CT is increasingly being used for follow-up. Evidence of progression of persistent disease, or of new disease after initial complete remission, should be biopsied, though this should be cautiously approached within 6 months after treatment completion, as tumor regression can continue to occur several months after the completion of chemoradiation.

\section{Treatment in HIV-positive populations}

As discussed above, the incidence of invasive anal cancer appears to be rising in the HIV-positive population, even in the highly active antiretroviral therapy era (HAART). 
It is believed the increased anal cancer risk is due to the high prevalence of HPV infection in this population. For example, anal HPV-16 was detectable in 35\% of HIV-positive men, compared with $13 \%$ of HIV-negative men in a recent meta-analysis of HPV and anal cancer in MSM. ${ }^{35}$ In this meta-analysis of 53 studies, the anal cancer incidence in the HIV-positive population was 45.9 per 100,000 men, compared with 5.1 per 100,000 men in the HIV-negative cohort.

Though there is no prospective randomized clinical trial, several single-institution series suggest that HIV-positive patients appear as likely to achieve complete remission as HIV-negative patients undergoing the same chemoradiation program. However, HIV-positive patients tend to have de-escalated chemotherapy and radiation, due to concerns about immunosuppression and increased treatment-related toxicity while on HAART. In fact, a retrospective single-institution study demonstrated that HIV-positive patients tended to have lower adherence to chemotherapy (30\% compared with $80 \%$ in HIV-negative patients), ${ }^{36}$ with reportedly increased rates of cisplatin substitution for MMC and dose modifications for concomitant medication, low CD4 count, or severe thrombocytopenia during treatment. This series also showed that local failure in the HIV-positive group was higher than in the HIVnegative group, possibly also negatively impacting colostomyfree survival, though this study is limited by small sample size. Overall survival appears to be the same between HIV-positive and HIV-negative patients in this and other single institution series. ${ }^{36-38}$ Current clinical practice recommendations are to treat HIV-positive patients at full chemotherapy and radiation dose, with added surveillance of possible treatment-related toxicities including dermatitis and hematologic toxicities.

\section{Experimental treatments}

Intensity-modulated radiation therapy (IMRT) is being increasingly used for multiple disease sites for dose escalation and/or minimization of high dose to surrounding normal tissue. IMRT can allow for improved coverage of both involved and at-risk inguinal and pelvic lymph nodes, while maintaining safe radiation doses to at-risk tissues such as the skin, rectum, pelvic bones, external genitalia, small bowel, and urethra. This represents an evolution from traditional radiation planning using large parallel-opposed anterior-posterior/posterior-anterior fields, in which there was significant acute skin, gastrointestinal, and genitourinary toxicity. This toxicity can delay treatment completion, is known to compromise treatment response, and can result in acute and long-term impairments in quality of life. Due to the ability to shape radiation beams around clinical target volumes with IMRT, toxicity to surrounding normal tissue can potentially be reduced, as reported in several institutional series. ${ }^{39-42}$ To date, IMRT has been best studied in a multi-institutional prospective phase II study, RTOG 05-29. Sixty-one patients with T2-4N0-3M0 anal canal cancer were evaluated with dose painted (DP)-IMRT prescribed as follows: T2N0: 42 Gy, 1.5 Gy/fraction to elective nodal planning target volume (PTV) and 50.4 Gy, 1.8 Gy/fraction to anal tumor PTV; T3-4N03: $45 \mathrm{~Gy}, 1.5 \mathrm{~Gy} /$ fraction to elective nodes, $50.4 \mathrm{~Gy}, 1.68$ Gy/fraction to involved lymph nodes $\leq 3 \mathrm{~cm}$ and 54 Gy to involved lymph nodes $>3 \mathrm{~cm}$, and 54 Gy to the anal tumor. Patients received 5-FU $\left(1000 \mathrm{mg} / \mathrm{m}^{2} /\right.$ day, 96 hour continuous infusion) and MMC (10 mg/m² IV bolus) on days 1 and 29 of IMRT. The primary endpoint of the study was to determine if combined $\geq$ grade 2 gastro intestional/genito unrinary (GI/GU) acute adverse events with DP-IMRT was decreased by $15 \%$ as compared to the RTOG 98-11 MMC arm. DP-IMRT yielded a statistically significant reduction in $\geq$ grade $3 \mathrm{GI} / \mathrm{GU}$ adverse events (22\% vs $36 \%$ in RTOG $98-11, P=0.014), \geq$ grade 2 dermatologic adverse events (69\% vs $81 \%$ in RTOG 98-11, $P=0.039$ ), and $\geq$ grade 3 dermatologic adverse events (20\% vs $47 \%$ in RTOG $98-11, P<0.0001) .{ }^{39}$ Additionally, preliminary outcomes demonstrate a 2-year locoregional failure rate of $20 \%$, 2-year colostomy rate of $8 \%$, and a 2-year disease-free survival rate of $77 \%{ }^{43}$ These results are similar to 2-year outcomes previously reported with conventional radiation in the 5-FU/MMC arm of RTOG 98-11.43 Additionally, a recent large multi-institutional retrospective series using IMRT with concurrent 5-FU/MMC reported acute grade 4 hematologic toxicity in $12.9 \%$ of patients, acute grade $\geq 3$ dermatological toxicity in $29.0 \%$ of patients, and acute grade $\geq 3$ gastrointestinal toxicity in $27.7 \%$ of patients. ${ }^{40}$ There were no reported grade 4 dermatologic nor gastrointestinal toxicities. In another retrospective, multiinstitutional study from Boston University and Massachusetts General Hospital, acute grade $\geq 3$ toxicities were $51 \%$ hematologic, 10\% dermatologic, 7\% gastrointestinal, and $7 \%$ genitourinary. ${ }^{41}$ These toxicity rates compared favorably to those reported in the MMC arm of RTOG 98-11, which did not allow IMRT. ${ }^{27,28}$ Because of the associated acute toxicity sparing, DP-IMRT has been incorporated as the radiation platform for future RTOG anal cancer trials, which may allow for radiation dose escalation.

Furthermore, there is interest in using targeted therapy in combination with traditional chemotherapy agents. Overexpression of the epidermal growth factor receptor 
(EGFR) in anal cancer has been demonstrated in several studies. ${ }^{44,45}$ Cetuximab, an EGFR inhibitor, shows promise in early results in metastatic anal cancer, ${ }^{46}$ and is being used in a Phase II trial with 5-FU/cisplatin and radiation in locally advanced cancer, following a Phase I trial in 10 patients that showed no dose-limiting toxicities to the regimen. ${ }^{47}$

Because of the significant association of anal cancer with HPV infection, further research is needed on the efficacy of the HPV vaccine in preventing anal cancer, and in using molecular genetics to characterize and perhaps stratify patients with high-risk HPV infections for more tailored treatment.

The search for potential predictive or prognostic biomarkers in anal cancer is another avenue of current research. TP53, Bcl-2, p21, and surviving are among the candidates for prognostic markers, though other studies have not consistently validated these results. ${ }^{48-50}$ Currently there are no biomarkers available for anal cancer.

\section{Conclusion and future directions}

Concurrent chemoradiation with 5-FU/mitomycin remains the standard of care for the treatment of anal cancer. There is currently no proven role for platinum-based induction or adjuvant chemotherapy in anal cancer, even in cases of bulky disease. Multiple trials have shown that radiosensitization with concurrent chemotherapy is beneficial over radiation alone, and in particular, efforts to remove or substitute mitomycin from the chemoradiation regimen have been unsuccessful. Because local-regional control remains a challenge in the management of anal cancer, future studies will need to focus on radiation dose-escalation and/or addition of further chemotherapy or targeted agents. Patient selection, eg, with PET-CT or with biomarkers including HPV status, may be necessary to define patients who need more aggressive local treatment, ie, for patients with bulky disease, or to de-escalate treatment in others, ie, patients with early-stage, localized cancer.

\section{Disclosure}

The authors report no conflicts of interest in this work.

\section{References}

1. SEER Cancer Statistics Review, 1975-2009 (Vintage 2009 Populations) [database on the Internet]. Bethesda, MD: National Cancer Institute. Available from http://seer.cancer.gov/csr/1975_2009_pops09/.Accessed November 6, 2012.

2. Sato H, Koh PK, Bartolo DC. Management of anal canal cancer. Dis Colon Rectum. 2005;48(6):1301-1315.

3. Frost DB, Richards PC, Monague ED, Giacco GG, Martin RG. Epidermoid cancer of the anorectum. Cancer. 1984;53(6):1285-1293.

4. Klotz RG Jr, Pamukcoglu T, Souilliard DH. Transitional cloacogenic carcinoma of the anal canal. Clinicopathologic study of three hundred seventy-three cases. Cancer. 1967;20(10):1727-1745.
5. Nigro ND, Vaitkevicius VK, Considine B Jr. Combined therapy for cancer of the anal canal: a preliminary report. Dis Colon Rectum. 1974;17(3):354-356.

6. Nigro ND, Seydel HG, Considine B, Vaitkevicius VK, Leichman L, Kinzie JJ. Combined preoperative radiation and chemotherapy for squamous cell carcinoma of the anal canal. Cancer. 1983;51(10): 1826-1829.

7. NCCN Clinical Practice Guidelines in Oncology. Fort Washington, PA: National Comprehensive Cancer Network; c2012. Available from: http://www.nccn.org. Accessed October 24, 2012.

8. Johnson LG, Madeleine MM, Newcomer LM, Schwartz SM, Daling JR. Anal cancer incidence and survival: the surveillance, epidemiology, and end results experience, 1973-2000. Cancer. 2004;101(2): 281-288.

9. Siegel R, Naishadham D, Jemal A. Cancer statistics, 2012. CA Cancer J Clin. 2012;62(1):10-29.

10. Chaturvedi AK, Madeleine MM, Biggar RJ, Engels EA. Risk of human papillomavirus-associated cancers among persons with AIDS. J Natl Cancer Inst. 2009;101(16):1120-1130.

11. Piketty C, Selinger-Leneman H, Grabar S, et al; for FHDH-ANRS CO 4. Marked increase in the incidence of invasive anal cancer among HIV-infected patients despite treatment with combination antiretroviral therapy. AIDS. 2008;22(10):1203-1211.

12. Daling JR, Madeleine MM, Johnson LG, et al. Human papillomavirus, smoking, and sexual practices in the etiology of anal cancer. Cancer. 2004;101(2):270-280.

13. Hoots BE, Palefsky JM, Pimenta JM, Smith JS. Human papillomavirus type distribution in anal cancer and anal intraepithelial lesions. Int $J$ Cancer. 2009;124(10):2375-2383.

14. Saboo SS, Zukotynski K, Shinagare AB, Krajewski KM, Ramaiya N. Anal carcinoma: FDG PET/CT in staging, response evaluation, and follow-up. Abdom Imaging. Epub September 26, 2012.

15. Mistrangelo M, Pelosi E, Bello M, et al. Role of positron emission tomography-computed tomography in the management of anal cancer. Int J Radiat Oncol Biol Phys. 2012;84(1):66-72.

16. Vercellino L, Montravers F, de Parades V, et al. Impact of FDG PET/ $\mathrm{CT}$ in the staging and the follow-up of anal carcinoma. Int J Colorectal Dis. 2011;26(2):201-210.

17. De Vuyst H, Clifford GM, Nascimento MC, Madeleine MM, Franceschi S. Prevalence and type distribution of human papillomavirus in carcinoma and intraepithelial neoplasia of the vulva, vagina and anus: A meta-analysis. Int J Cancer. 2009;124(7):1626-1636.

18. Gillison ML, Chaturvedi AK, Lowy DR. HPV prophylactic vaccines and the potential prevention of noncervical cancers in both men and women. Cancer. 2008;113(Suppl 10):3036-3046.

19. Palefsky JM, Giuliano AR, Goldstone S, et al. HPV vaccine against anal HPV infection and anal intraepithelial neoplasia. $N$ Engl J Med. 2011;365(17):1576-1585.

20. Swedish KA, Factor SH, Goldstone SE. Prevention of recurrent highgrade anal neoplasia with quadrivalent human papillomavirus vaccination of men who have sex with men: a nonconcurrent cohort study. Clin Infect Dis. 2012;54(7):891-898.

21. Epidermoid anal cancer: results from the UKCCCR randomised trial of radiotherapy alone versus radiotherapy, 5-fluorouracil, and mitomycin. UKCCCR Anal Cancer Trial Working Party. UK Co-ordinating Committee on Cancer Research. Lancet. 1996;348(9034):1049-1054.

22. Bartelink H, Roelofsen F, Eschwege F, et al. Concomitant radiotherapy and chemotherapy is superior to radiotherapy alone in the treatment of locally advanced anal cancer: results of a phase III randomized trial of the European Organization for Research and Treatment of Cancer Radiotherapy and Gastrointestinal Cooperative Groups. J Clin Oncol. 1997;15(5):2040-2049.

23. Flam M, John M, Pajak TF, et al. Role of mitomycin in combination with fluorouracil and radiotherapy, and of salvage chemoradiation in the definitive nonsurgical treatment of epidermoid carcinoma of the anal canal: results of a phase III randomized intergroup study. J Clin Oncol. 1996;14(9):2527-2539. 
24. Chakravarthy AB, Catalano PJ, Martenson JA, et al. Long term follow-up of a phase II trial of high-dose radiation with concurrent 5-fluorouracil and cisplatin in patients with anal cancer (ECOG E4292). Int J Radiat Oncol Biol Phys. 2011;81(4):e607-e613.

25. Peiffert D, Giovannini M, Ducreux M, et al; for Digestive Tumours Group of the French 'Fédération Nationale des Centres de Lutte Contre le Cancer'. High-dose radiation therapy and neoadjuvant plus concomitant chemotherapy with 5-fluorouracil and cisplatin in patients with locally advanced squamous-cell anal canal cancer: final results of a phase II study. Ann Oncol. 2001;12(3):397-404.

26. Meropol NJ, Niedzwiecki D, Shank B, et al. Induction therapy for poor-prognosis anal canal carcinoma: a phase II study of the cancer and Leukemia Group B (CALGB 9281). J Clin Oncol. 2008;26(19): 3229-3234.

27. Ajani JA, Winter KA, Gunderson LL, et al. Fluorouracil, mitomycin, and radiotherapy vs fluorouracil, cisplatin, and radiotherapy for carcinoma of the anal canal: a randomized controlled trial. JAMA. 2008; 299(16):1914-1921

28. Gunderson LL, Winter KA, Ajani JA, et al. Long-term update of US GI Intergroup RTOG 98-11 phase III trial for anal carcinoma: Survival, relapse and colostomy failure with concurrent chemoradiation involving 5FU-mitomycin versus 5FU-cisplatin. J Clin Oncol. In press 2012.

29. Ben-Josef E, Moughan J, Ajani JA, et al. Impact of overall treatment time on survival and local control in patients with anal cancer: a pooled data analysis of Radiation Therapy Oncology Group trials 87-04 and 98-11. J Clin Oncol. 2010;28(34):5061-5066.

30. Graf R, Wust P, Hildebrandt B, et al. Impact of overall treatment time on local control of anal cancer treated with radiochemotherapy. Oncology. 2003;65(1):14-22.

31. James R, Wan S, Glynne-Jones R, et al; and National Cancer Research Institute (NCRI) ACT II Trial Management Group, Maidstone General Hospital, Cancer Research UKand UCL Cancer Trials Centre et al. A randomized trial of chemoradiation using mitomycin or cisplatin, with or without maintenance cisplatin/5-FU in squamous cell carcinoma of the anus (ACT II) [abstract]. J Clin Oncol. 2009;27 Suppl 18:LBA4009.

32. Glynne-Jones R, James R, Meadows H, et al. Optimum time to assess complete clinical response (CR) following chemoradiation (CRT) using mitomycin (MMC) or cisplatin (CisP), with or without maintenance CisP/5FU in squamous cell carcinoma of the anus: Results of ACT II [abstract]. J Clin Oncol. 2012;30 Suppl:Abstr 4004.

33. Olivatto LO, Cabral V, Rosa A, et al. Mitomycin-C- or cisplatin-based chemoradiotherapy for anal canal carcinoma: long-term results. Int $J$ Radiat Oncol Biol Phys. 2011;79(2):490-495.

34. Peiffert D, Tournier-Rangeard L, Gérard JP, et al. Induction chemotherapy and dose intensification of the radiation boost in locally advanced anal canal carcinoma: final analysis of the randomized UNICANCER ACCORD 03 trial. J Clin Oncol. 2012;30(16):1941-1948.

35. Machalek DA, Poynten M, Jin F, et al. Anal human papillomavirus infection and associated neoplastic lesions in men who have sex with men: a systematic review and meta-analysis. Lancet Oncol. 2012; 13(5):487-500.

36. Oehler-Jänne C, Seifert B, Lütolf UM, Ciernik IF. Local tumor control and toxicity in HIV-associated anal carcinoma treated with radiotherapy in the era of antiretroviral therapy. Radiat Oncol. 2006;1:29.
37. Chiao EY, Giordano TP, Richardson P, El-Serag HB. Human immunodeficiency virus-associated squamous cell cancer of the anus: epidemiology and outcomes in the highly active antiretroviral therapy era. J Clin Oncol. 2008;26(3):474-479.

38. Hammad N, Heilbrun LK, Gupta S, et al. Squamous cell cancer of the anal canal in HIV-infected patients receiving highly active antiretroviral therapy: a single institution experience. Am J Clin Oncol. 2011;34(2):135-139.

39. Kachnic LA, Winter KA, Myerson RJ, et al. RTOG 0529: A phase II study of dose-painted IMRT (DP-IMRT), 5-fluorouracil, and mitomycin- $\mathrm{C}$ for the reduction of acute morbidity in anal cancer [abstract]. 2010 ASCO Gastrointestinal Cancers Symposium, 2010 January 22-24; Orlando, USA, abstract 450.

40. DeFoe SG, Beriwal S, Jones H, et al. Concurrent Chemotherapy and Intensity-modulated Radiation Therapy for Anal Carcinoma Clinical Outcomes in a Large National Cancer Institute-designated Integrated Cancer Centre Network. Clin Oncol ( $R$ Coll Radiol). 2012;24(6):424-431.

41. Kachnic LA, Tsai HK, Coen JJ, et al. Dose-painted intensity-modulated radiation therapy for anal cancer: a multi-institutional report of acute toxicity and response to therapy. Int J Radiat Oncol Biol Phys. 2012;82(1):153-158.

42. Brooks CJ, Lee YK, Aitken K, Hansen VN, Tait DM, Hawkins MA Organ-sparing Intensity-modulated Radiotherapy for Anal Cancer using the ACTII Schedule: A Comparison of Conventional and Intensitymodulated Radiotherapy Plans. Clin Oncol (R Coll Radiol). Epub September 14, 2012.

43. Kachnic LA, Winter KA, Myerson RJ, et al. Two-year outcomes of RTOG 0529: A phase II evaluation of dose-painted IMRT in combination with 5-fluorouracil and mitomycin- $\mathrm{C}$ for the reduction of acute morbidity in carcinoma of the anal canal [abstract]. J Clin Oncol. 2011;29 Suppl 4:368.

44. Alvarez G, Perry A, Tan BR, Wang HL. Expression of epidermal growth factor receptor in squamous cell carcinomas of the anal canal is independent of gene amplification. Mod Pathol. 2006;19(7):942-949.

45. Lê LH, Chetty R, Moore MJ. Epidermal growth factor receptor expression in anal canal carcinoma. Am J Clin Pathol. 2005;124(1): 20-23.

46. Lukan N, Ströbel P, Willer A, et al. Cetuximab-based treatment of metastatic anal cancer: correlation of response with KRAS mutational status. Oncology. 2009;77(5):293-299.

47. Olivatto LO, Araujo CM, Vilhena B, et al. Phase I study of cetuximab (CET) in combination with 5-FU, cisplatin (CP), and radiotherapy (RT) in patients with locally advanced squamous cell anal canal carcinoma (LASACC) [abstract]. 2010 Gastrointestinal Cancers Symposium; January 22-24, 2010; Orlando, FL.

48. Allal AS, Waelchli L, Bründler MA. Prognostic value of apoptosisregulating protein expression in anal squamous cell carcinoma. Clin Cancer Res. 2003;9(17):6489-6496.

49. Ajani JA, Wang X, Izzo JG, et al. Molecular biomarkers correlate with disease-free survival in patients with anal canal carcinoma treated with chemoradiation. Dig Dis Sci. 2010;55(4):1098-1105.

50. Fraunholz I, Rödel C, Distel L, et al. High survivin expression as a risk factor in patients with anal carcinoma treated with concurrent chemoradiotherapy. Radiat Oncol. 2012;7:88.

Gastrointestinal Cancer: Targets and Therapy

\section{Publish your work in this journal}

Gastrointestinal Cancer: Targets and Therapy is an international, peer-reviewed, open access journal focusing on gastro-intestinal cancer research, identification of therapeutic targets and the optimal use of preventative and integrated treatment interventions to achieve improved outcomes, enhanced survival and quality of life for the

cancer patient. The manuscript management system is completely online and includes a very quick and fair peer-review system. Visit http://www.dovepress.com/testimonials.php to read real quotes from published authors. 